\title{
Characteristics of the interaction in azulene $\cdots\left(H_{2} X\right)_{n=1,2} \quad(X=O, S)$ clusters.
}

Enrique M. Cabaleiro-Lago ${ }^{(a)}$, Ángeles Peña-Gallego ${ }^{(b)}$, Jesús Rodríguez-Otero ${ }^{(b)}$, M. Merced Montero-Campillo ${ }^{(b)}$

(a) Departamento de Química Física, Facultade de Ciencias, Universidade de Santiago de Compostela, Campus de Lugo. Avda. Alfonso XEl Sabio s/n 27002 Lugo, Galicia (Spain).

(b) Departamento de Química Física, Facultade de Química, Universidade de Santiago de Compostela, Avda. das Ciencias, s/n 15706 Santiago de Compostela, Galicia (Spain).

\section{e-mail: qftkike@usc.es}

\begin{abstract}
A computational study of clusters containing azulene and up to two molecules of water or hydrogen sulfide was carried out to elucidate the main characteristics of these $\mathrm{X}-\mathrm{H} \cdots \pi$ interacting systems. For clusters with one $\mathrm{H}_{2} \mathrm{X}$ molecule only one structure was found interacting with the aromatic cloud of azulene, with an interaction energy of $-3.1 \mathrm{kcal} / \mathrm{mol}$ both for $\mathrm{H}_{2} \mathrm{O}$ and $\mathrm{H}_{2} \mathrm{~S}$ as calculated at the CCSD(T)/AVDZ level. On the other hand, MP2 overestimates the interaction in hydrogen sulfide clusters, whereas the MPWB1K functional produces values in very good agreement with $\operatorname{CCSD}(\mathrm{T})$.

A variety of structures were located for clusters with two $\mathrm{H}_{2} \mathrm{X}$ molecules. The most stable ones are those which simultaneously present hydrogen bond between $\mathrm{H}_{2} \mathrm{X}$ molecules and $\mathrm{X}-\mathrm{H} \cdots \pi$ contacts. Also, only this kind of structure presents relevant three body stabilizing contributions.

On the other hand, the interaction of azulene with $\left(\mathrm{H}_{2} \mathrm{X}\right)_{2}$ dimer is stronger precisely for structures which do not present $\mathrm{X}-\mathrm{H} \cdots \mathrm{X}$ hydrogen bond. This suggest that for larger systems, structures with the molecules distributed over the aromatic surface but without interacting among them, can be competitive with other, hydrogen bonded clusters, especially in $\mathrm{H}_{2} \mathrm{~S}$ containing systems.
\end{abstract}




\section{Introduction}

Intermolecular interactions involving systems containing aromatic molecules are of great importance in diverse fields. If the interaction involves the $\pi$ aromatic system, it is usually one of the three following types: cation $\cdots \pi ; \pi \cdots \pi$ or X-H $\cdots \pi$, which have been a subject of much recent interest. ${ }^{[1]}$ In the present work we have centered our attention in this last type of interaction, in which a delicate balance between different contributions settles down the interaction energy.

One type of interaction that has not received as much attention from a computational point of view is the sulfur- $\pi$ interaction, partly since it is not as common as the other in natural systems but also because the presence o the sulfur atom increases the computational expense. Also, for sulfur containing systems, it has been shown that the basis effects are more important than in oxygen containing analogs, so larger basis sets and high level calculation methods must be employed. ${ }^{[2]}$

Most studies of this kind have been carried out employing benzene as a model for aromatic systems, but there exists a lack of studies employing larger, poliaromatic systems. To our knowledge, only indole has been considered as a possible candidate for these studies since it forms part of triptophan. ${ }^{[3,4]}$ However, interactions with other policyles can be of interest in different areas, ranging from molecular recognition, because naphthalene and other units are employed as a constituent part of molecular tweezers, to cromatography, since this kind of interactions can be employed for separating policyclic aromatic hydrocarbons.

Therefore, in the present work the interaction in systems containing an azulene molecule and one or two water or hydrogen sulfide molecules was computationally studied. This kind of study allows to determine the main characteristics of the interaction with the aromatic cloud of these systems, as well as the balance between $\mathrm{C}_{10} \mathrm{H}_{8} \cdots \mathrm{H}_{2} \mathrm{X}$ and $\mathrm{H}_{2} \mathrm{X} \cdots \mathrm{H}_{2} \mathrm{X}$ interactions. Also, a comparison between water and hydrogen sulfide clusters allows revealing the main differences of the interaction with hydroxyl and thiol containing molecules. 


\section{Computational details and procedure}

Starting structures were constructed attending to chemical intuition, trying to represent the possible $\mathrm{X}-\mathrm{H} \cdots \pi$ favorable contacts. Several initial structures were therefore fully optimized by using the MP2 method together with the 6-31+G* basis set. For systems of the size of those studied in this work, and having in mind possible extension to even larger policycles, it is interesting to test the performance of DFT methods for this kind of interaction. After preliminary tests, a widely used functional as B3LYP reveals significant differences in structures when compared to MP2. This is not surprising since in previous works, several authors have claimed for the incorrect behavior of B3LYP in X-H $\cdots \pi$ interactions. ${ }^{[4,5]}$ In fact, the main problem arises from the large dispersive contribution to interaction energy, which has been a goal for functional developers. Recently, several functionals have been proposed for studying this kind of interaction. Among them, we opted to employ the MPWB1K functional proposed by Thrular's group as it is recommended as a well balanced functional with a good average performance in different kinds of systems, including dispersive ones. ${ }^{[6]}$ Also, previous calculations in indole-water complexes have shown that a similar functional gives good results. ${ }^{[4]}$ Therefore, the geometries of the complexes were also optimized with the MPWB1K functional together with the $6-31+\mathrm{G}^{*}$ basis set. All points were characterized as minima by calculating the harmonic vibrational frequencies.

After locating the stationary points of the potential energy surface of each cluster and having characterized them as minima by performing a vibrational analysis, the interaction energies where calculated by means of the counterpoise method to avoid basis set superposition error. Thus, the interaction energy results from subtracting the energies of the fragments that constitute the clusters employing the geometry and the whole basis set of the cluster ${ }^{[7,8]}$

$$
\Delta E_{\text {int. }}=E_{i j}(i j \ldots)-\sum_{i} E_{i}^{c l u s .}(i j \ldots)
$$

As the geometry of the molecules changes when the cluster is formed, and additional contribution describing this effect must be included, obtained as the energy difference between the molecules in the cluster geometry and in isolation.

$$
E_{\text {def. }}=\sum_{i}\left(E_{i}^{\text {clus. }}(i)-E_{i}^{\text {isol. }}(i)\right)
$$


The total complexation energy results from adding these two contributions, though deformation effects are usually small and negligible for most clusters, and in the discussion we will not consider them.

To estimate the basis set effects, interaction energies were also computed by employing a larger basis set: aug-cc-pvdz in heavy atoms and cc-pvdz in hydrogen atoms (henceforth AVDZ). Finally, selected high level computations were performed; that is, interaction energies of complexes with one $\mathrm{H}_{2} \mathrm{X}$ molecule were obtained at CCSD(T)/AVDZ level.

A deeper analysis of the interaction has been carried out in the case of complexes with two $\mathrm{H}_{2} \mathrm{X}$ molecules by calculating interaction energies for each pair of molecules that constitute the cluster. This analysis also allows estimating three body effects.

Supermolecule method gives a plain number as result, so a perturbational analysis was carried out to have more insight into the nature of the interaction. A Symmetry Adapted Perturbation Theory (SAPT) analysis was performed to compute the different contributions to the interaction energy. In the case of trimers, the analysis was performed for the interaction between azulene and $\mathrm{H}_{2} \mathrm{X}$ dimer. The calculations were carried out by employing the SAPT-DFT approach, which has been shown to provide reasonable estimation of interaction energy contributions at a lower computational cost than ordinary SAPT. These calculations were performed by using the SAPT2006 code of Szalewicz and coworkers ${ }^{[9]}$ interfaced to Dalton 2.0 package. ${ }^{[10]}$ All other calculations were performed with Gaussian03. ${ }^{[11]}$ 


\section{Results}

Figure 1 shows the minimum energy structures found for the complexes with one $\mathrm{H}_{2} \mathrm{X}$ molecule. It can be appreciated that both structures are pretty similar, with the $\mathrm{H}_{2} \mathrm{X}$ molecule above the aromatic cloud. However, whereas in the water complex, water molecule is clearly displaced to the five carbon ring, the hydrogen sulfide molecule is located more symmetrically over the rings, though slightly nearer the seven carbon ring. The results are consistent with those observed in indole, where no water complex was found with the water over the larger ring. ${ }^{[3,4]}$ As regards the methods employed in geometry optimizations, no significant differences were found between MP2 and MPWB1K.

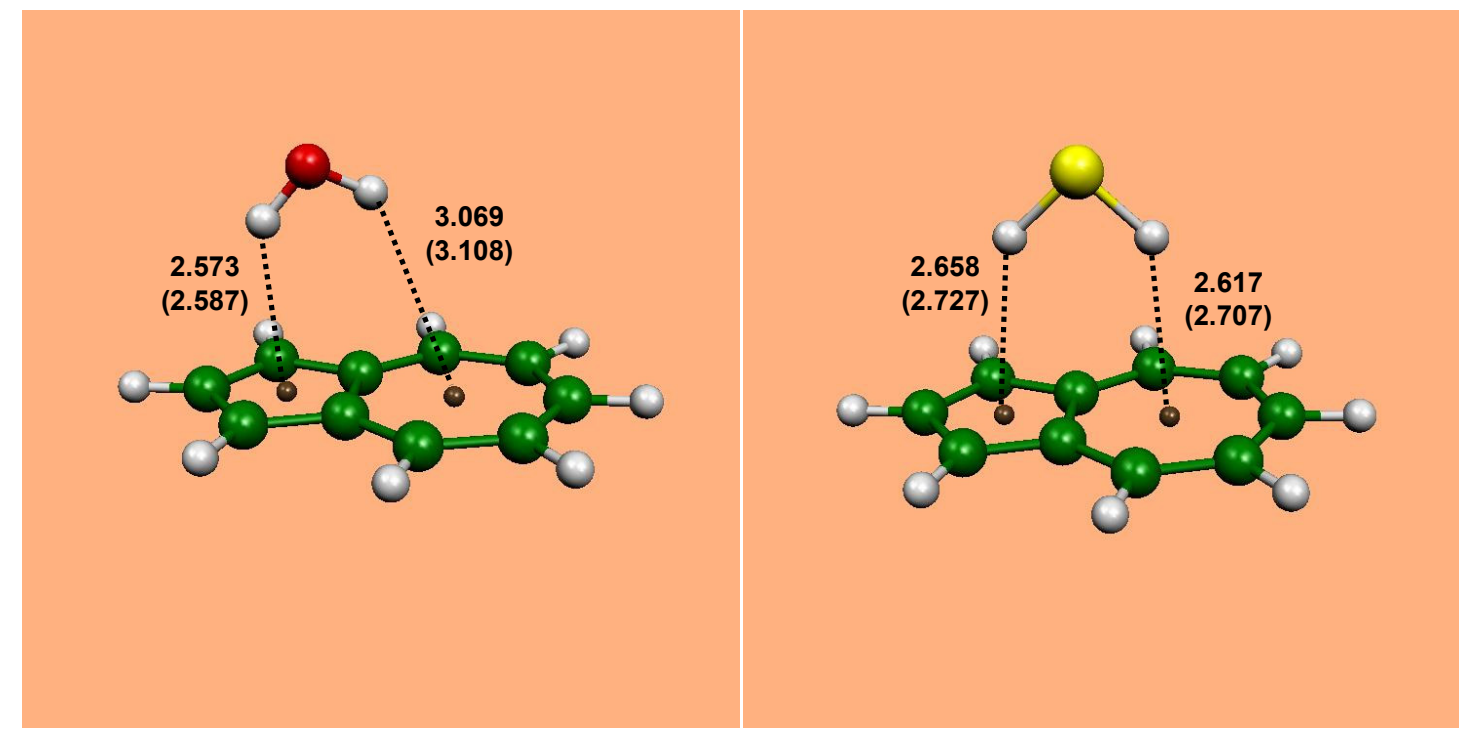

Figure 1. Minimum energy structures located for the clusters with one $\mathrm{H}_{2} \mathrm{X}$ molecule. Distances to the center of the rings in $\AA$. MP2/6-31+G* values; MPWB1K values in parentheses. 
Table 1 lists the interaction energies computed at different levels. It can be observed that MP2 and MPWB1K give similar results when employed together with the $6-31+\mathrm{G}^{*}$ basis set, though the DFT method produces somewhat larger interaction energies. Increasing the size of the basis set has important effects in MP2 results. Thus, interaction energies changes by almost $-1 \mathrm{kcal} / \mathrm{mol}$ in the case of water complex and $-2 \mathrm{kcal} / \mathrm{mol}$ in the case of the hydrogen sulfide one. On the other hand, DFT results remain unchanged when basis set is enlarged. Though the observed behavior can be associated to a better recovery of dispersion interaction by MP2 method, it has been claimed that MP2 results overestimate dispersive interactions, so the increase in interaction energy could be a problem of the method employed. To check this, coupled cluster calculations were carried out. CCSD gives results similar to those obtained with the smaller basis set. Inclusion of triples increases the intensity of the interaction, which reaches about $-3 \mathrm{kcal} / \mathrm{mol}$ for both complexes, in very good agreement with MPWB1K results. Comparison with results obtained for the interaction of benzene and water or hydrogen sulfide obtained at similar levels of calculations, indicates that the interaction wit azulene has similar intensity. ${ }^{[2,12]}$

Table 1. Interaction energies $(\mathrm{kcal} / \mathrm{mol})$ for the clusters with one $\mathrm{H}_{2} \mathrm{X}$ molecule computed with different methods.

\begin{tabular}{|l|c|c|}
\hline & $\mathbf{H}_{2} \mathbf{O}$ & $\mathbf{H}_{2} \mathbf{S}$ \\
\hline MPWB1K/6-31+G & -3.22 & -2.93 \\
\hline MP2/6-31+G* & -2.76 & -2.63 \\
\hline MP2/AVDZ // MP2/6-31+G* & -3.58 & -4.62 \\
\hline MPWB1K/AVDZ // MPWB1K/6-31+G & -3.09 & -2.94 \\
\hline CCSD/AVDZ // MP2/6-31+G* & -2.70 & -2.38 \\
\hline CCSD(T)/AVDZ // MP2/6-31+G* & -3.09 & -3.08 \\
\hline
\end{tabular}


In the case of complexes with two $\mathrm{H}_{2} \mathrm{X}$ molecules the situation is more difficult, since several minimum energy structures where located with both MP2 and MPWB1K methods. These structures are shown in Figure 2 together with the most relevant distances.

Two of the structures (Azu-B and Azu-C) can be considered as pure $\pi$ interacting structures whereas the other one (Azu-A) presents deviated sigma interactions with one of the $\mathrm{X}$ atoms acting as acceptor. It is worth noting that structure Azu-B does not present hydrogen bond between $\mathrm{H}_{2} \mathrm{X}$ molecules and probably will be the less stable of these structures. However it could represent a situation where molecules with low tendency to association are spread over a large poliaromatic molecule

Table 2. Interaction energy and energy decomposition by pairs $(\mathrm{kcal} / \mathrm{mol})$ for the clusters with two $\mathrm{H}_{2} \mathrm{X}$ molecules. MPWB1K/AVDZ//MPWB1K/6-31+G*. 1 and 2 are $\mathrm{H}_{2} \mathrm{X}$ molecules.

\begin{tabular}{|c|c|c|c|c|c|c|c|}
\hline & & $\mathbf{H}_{\mathbf{2}} \mathbf{O}$ & & & & $\mathbf{H}_{2} \mathbf{S}$ & \\
\hline & $\mathbf{A z u}-\mathbf{A}$ & $\mathbf{A z u}-\mathbf{B}$ & $\mathbf{A z u}-\mathbf{C}$ & & $\mathbf{A z u}-\mathbf{A}$ & $\mathbf{A z u}-\mathbf{B}$ & Azu-C \\
\hline $\mathbf{A z u}+\mathbf{1 + 2}$ & -10.32 & -5.54 & -9.21 & & -5.92 & -5.40 & -4.26 \\
\hline $\mathbf{1 + 2}$ & -4.56 & 0.33 & -3.49 & & -1.10 & 0.00 & -1.04 \\
\hline $\mathbf{A z u}+\mathbf{1}$ & -1.75 & -3.04 & -2.88 & & -1.38 & -2.83 & -1.88 \\
\hline $\mathbf{A z u}+\mathbf{2}$ & -2.49 & -2.96 & -2.39 & & -2.77 & -2.83 & -1.22 \\
\hline Sum-pair & -8.80 & -5.67 & -8.76 & & -5.25 & -5.66 & -4.14 \\
\hline Nopair & -1.52 & 0.13 & -0.45 & & -0.68 & 0.26 & -0.12 \\
\hline Azu+(1-2) & -5.76 & -5.87 & -5.71 & & -4.83 & -5.40 & -3.22 \\
\hline
\end{tabular}



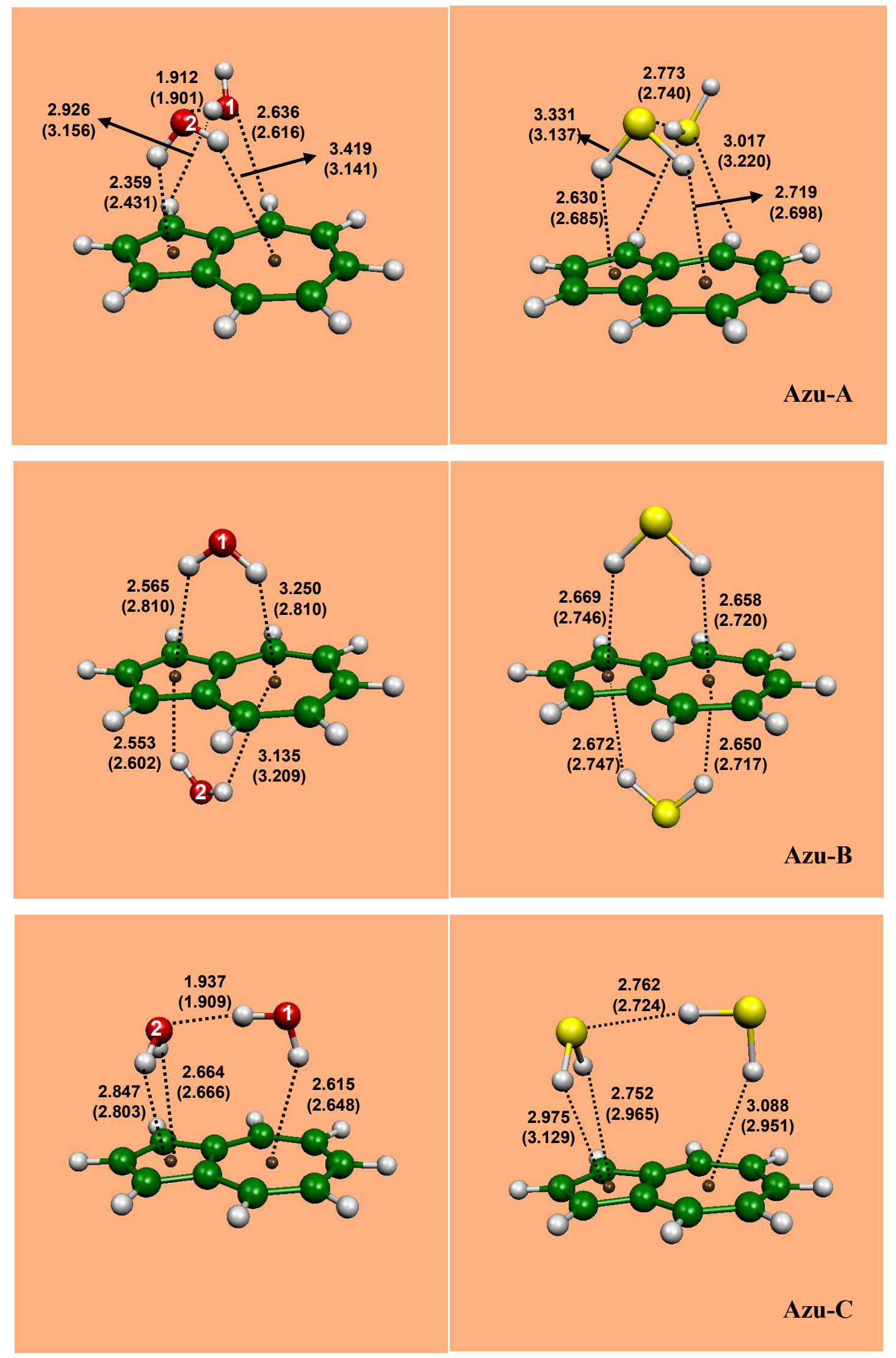

Figure 2. Minimum energy structures found for azulene $\cdots\left(\mathrm{H}_{2} \mathrm{X}\right)_{2}$ clusters. Distances in $\AA$. MP2/6-31G* values; MPWB1K values in parentheses. 
As regards interaction energies, these are shown in Table 2. Taking into account the results obtained for clusters with one $\mathrm{H}_{2} \mathrm{X}$ molecule, only MPWB1K/AVDZ results are presented. Besides interaction energies, a decomposition analysis was performed by calculating the pair interaction energies for each three molecule cluster. Also, the interaction energy between azulene and the $\mathrm{H}_{2} \mathrm{X}$ dimer was also computed.

It can be inferred from Table 2, that in water clusters the most stable structure corresponds to the so-called Azu-A structure, where a hydrogen bond is present between water molecules. The second most stable minimum corresponds to a similar structure, Azu-C, where the hydrogen bond is present, but in this case water molecules point downwards to the aromatic ring. This structure is less stable by $1.1 \mathrm{kcal} / \mathrm{mol}$. Finally structure Azu-B is much less attractive than in the other two due to the lack of $\mathrm{O}-\mathrm{H} \cdots \mathrm{O}$ hydrogen bond.

Considering the pair interaction analysis, the following tendencies can be observed. In structures Azu-B and Azu-C water molecules can interact almost freely with azulene, contributing to the interaction energy with an amount similar to that observed for the one molecule cluster. However, in the most stable structure, the interaction between water molecules and azulene is less favorable, but the interaction between water molecules is much stronger otherwise. It is also worth noting that the only structure where three-body interactions are relevant is Azu-A, associated to the hydrogen bond chain, and key for making this structure the most stable one. Finally, the interaction between azulene and the water dimer is also analysed. In this case, all structures behave similarly. Therefore, the order of stability depends on the water-water interaction, as in all structures the interaction with azulene is of similar magnitude.

Similar behavior is observed for $\mathrm{H}_{2} \mathrm{~S}$ clusters though, in this case, the weaker hydrogen bond interaction between hydrogen sulfide molecules produces structures very similar in stability. Thus, considering pair interactions, all structures present similar interaction energies, and Azu-A is the most stable due to three-body effects, which are present but in smaller magnitude than in water clusters. Finally, the interaction between azulene and the $\mathrm{H}_{2} \mathrm{~S}$ dimer is strongest for Azu-B. This result, together with the similar interaction energy of Azu-B as compared with other structures suggest that it is possible that for larger policycles the $\mathrm{H}_{2} \mathrm{~S}$ molecules tend to distribute over aromatic rings, prevailing the $\mathrm{H}_{2} \mathrm{~S} \cdots \pi$ interaction over the tendency to form hydrogen bonds between $\mathrm{H}_{2} \mathrm{~S}$ molecules. 
Figure 3 shows energy decomposition by using SAPT method for all clusters studied in the MP2 optimized structure. The main difference between water and hydrogen sulfide clusters relies, apart from a larger repulsion in $\mathrm{H}_{2} \mathrm{~S}$ clusters, in the dispersive contribution, which is much larger in hydrogen sulfide containing systems. Thus, in clusters containing one $\mathrm{H}_{2} \mathrm{X}$ molecule, the dispersion interaction contributes $1.5 \mathrm{kcal} / \mathrm{mol}$ more in hydrogen sulfide cluster, and this behavior is reproduced in clusters with two $\mathrm{H}_{2} \mathrm{X}$ molecules, the differences being even larger. It is also worth noting that, as shown in the pair energy decomposition of Table 2 , non additive effects are absent in structures Azu-B. Therefore, all contributions correspond in a good approximation to twice the values observed in the cluster with one $\mathrm{H}_{2} \mathrm{X}$ molecule.
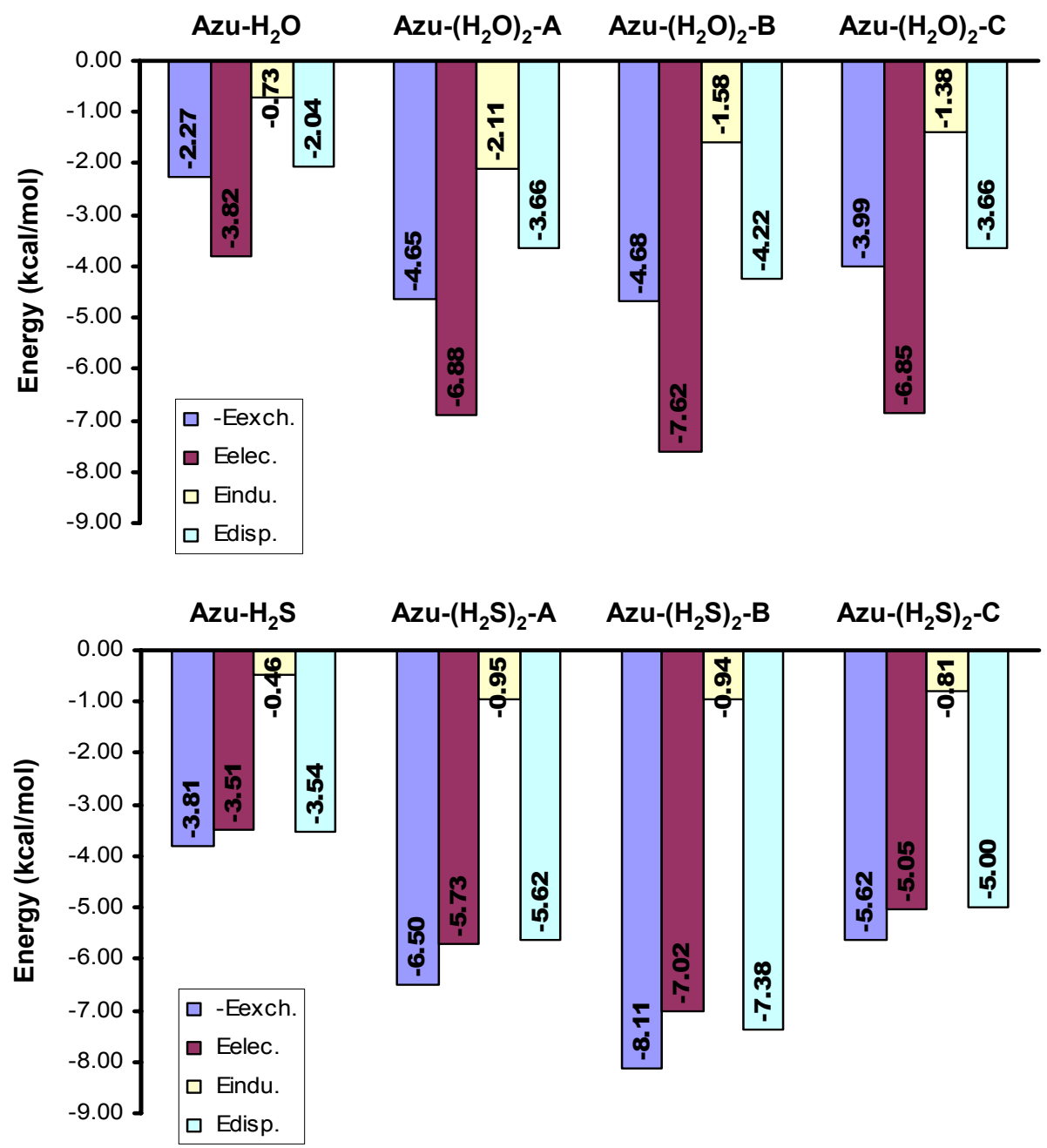

Figure 3. SAPT energy decomposition analysis $(\mathrm{kcal} / \mathrm{mol})$ for the clusters studied. For $\mathrm{Azu} \cdots\left(\mathrm{H}_{2} \mathrm{X}\right)_{2}$ clusters the analysis refers to the interactions between the $\left(\mathrm{H}_{2} \mathrm{X}\right)_{2}$ dimer and azulene molecule. 


\section{References}

(1) E. A. Meyer, R. K. Castellano, F. Diederich, Angew. Chem., Int. Ed. 2003, 42, 1210 .

(2) T. P. Tauer, M. E. Derrick, C. D. Sherrill, J. Phys. Chem. A 2005, 109, 191.

(3) T. van Mourik, S. L. Price, D. C. Clary, Chem. Phys. Lett. 2000, 331, 253.

(4) R. B. Zhang, K. R. F. Somers, E. S. Kryachko, M. T. Nguyen, T. ZeegersHuyskens, A. Ceulemans, J. Phys. Chem A 2005, 109, 8028.

(5) T. van Mourik, Chem. Phys. 2004, 304, 317.

(6) Y. Zhao, D. G. Truhlar, J. Chem. Theor. Comput. 2005, 1, 415.

(7) E. M. Cabaleiro-Lago, J. M. Hermida-Ramon, J. Rodríguez-Otero, J. Phys. Chem. A 2004, 108, 4923.

(8) S. F. Boys, F. bernardi, Mol. Phys. 1970, 18, 553.

(9) R. Bukowski et al. SAPT2006: An Ab Initio Program for Many-Body Symmetry-Adapted Perturbation Theory Calculations of Intermolecular Interaction Energies. Sequential and parallel versions.

See http://www.physics.udel.edu/ szalewic/SAPT/index.html

(10) DALTON, a molecular electronic structure program, Release 2.0 (2005), see http://www.kjemi.uio.no/software/dalton/dalton.html ".

(11) M. J. Frisch et al., Gaussian 03, Revision C.02. Gaussian, Inc., Wallingford CT, 2004 .

(12) S. Tsuzuki, K. Honda, T. Uchimaru, M. Mikami, K. Tanabe, J. Am. Chem. Soc. 2000, 122,11450 . 